\title{
Analysis of Guqin Music Transmission and Inheritance based on Network Environment
}

\author{
Ying Wang ${ }^{1}$, Wei Zhou ${ }^{2}$ \\ ${ }^{1}$ Jiangxi Science \& Technology Normal University, Jiangxi Province, Nanchang, 330013 \\ ${ }^{2}$ Nanchang Normal University, Jiangxi Province, Nanchang, 330032
}

Keywords: Network Environment; Guqin Music; Communication; Inheritance

\begin{abstract}
With the advent of the Internet age, the transmission and inheritance of guqin music have undergone significant changes, and there are significant differences in the propagation effect. Open free-style network communication, so that the music of the guqin music has been more people love, but the cultural characteristics also will be changed. Specialization tends to be popular, aesthetic to technical and introspection is also gradually replaced by entertainment. This paper first summarizes the characteristics of the transmission of guqin music in the background of the network, and then studies the influence of the change of the communication environment on the inheritance mode of the guqin music. Finally, it analyzes the pros and cons of the transmission of the guqin music in the background of the network. Sustainable development has the theoretical significance and practical significance which cannot be ignored.
\end{abstract}

\section{Introduction}

Undeniably, the rapid development of network information technology in this century has had a tremendous impact on the spread of traditional Chinese music culture. The new media of this network has changed the transmission and inheritance of guqin music. Although the different patterns of transmission, guqin culture show different characteristics, but many new problems are endless. Therefore, before analyzing the transmission of guqin music, we must understand the environmental characteristics of modern traditional music network communication, and as a starting point to study the network environment, the advantages and disadvantages of guqin music transmission and inheritance.

\section{The Transmission Characteristics of Guqin Music in the Network Context}

As with the traditional music special website, guqin music website also includes the official, civil and university of these three different types. At present, the most famous and official background of the guqin communication site is "Chinese guqin music network". In this site, including the organizational structure and charter, not only involves the guqin music itself, but also includes many social activities, such as awards, examinations, etc., authoritative and official.

The folk guqin music website mainly includes the integrated network of pilgrims and personal web pages; piano community of civil society set up a comprehensive website, guqin music lovers set up the site. Which is more typical of the Ding Ji Park hosted the "Chinese Guqin network." Most of this type of site focused on guqin music or music culture itself, with a strong pertinence [1].

The existence of the background of the independence of the guqin site is rare, usually built on the portal site blog or other community network, such as located in Guangdong Province, Sun Yat-sen University is to rely on "Sina blog" built of. This type of network spread because the audience is mainly concentrated in the members of the piano, so compared with the first two kinds of communication, the audience is still relatively small, the main form of content is the blog article, and then expand the exchange or release activities information. 


\section{The Impact of Communication Environment Changes on the Inheritance Method of Guqin Music}

Traditional guqin music inheritance there are two main, one is the folk heritage, the other is the school education heritage. And the inheritance of folk inheritance there are three main types of performance methods, respectively, ancestral, piano club heritage and piano heritage. Although in history, these traditional inheritance methods have a leading role in cultural communication, but the purpose of communication is always interpersonal communication, and the mass media, the depth and breadth of its spread is obviously insufficient. According to the principle of communication, network communication has the characteristics of remoteity, immediacy and delay.

On the one hand, the past "oral counseling" and school teaching, are interpersonal communication between teachers and students, will be severely restricted by space venues, so the number of learners is very limited. But in the network environment, will not be bound by the venue, to conduct distance education. Effectively make up for the narrow surface of the communication and the small size of the audience shortcomings.

On the other hand, the dynamic network of virtual space because of the powerful and wide range of practical, effectively promote the development of real-time teaching. In the network environment teaching, teachers and learning in the first time to communicate piano, gesture and language and other aspects, breaking the static network video cannot be flaws, for the guqin network heritage to open up a broader space. In addition, guqin music network inheritance also has a strong delay. That is, the audience can search for resources on the network without restrictions on the exchange of discussion, which effectively broke the traditional inheritance model of time and human constraints. Online community and the major guqin music sites are equipped with this feature. When the communicator publishes a question on the web, the audience can participate immediately in the discussion. If the guqin learners have a strong interest in the content of the study, or do not understand, you can search online, and soon be able to find the appropriate learning resources [2].

\section{The Pros and Cons of the Transmission of Guqin Music in the Network Background}

The Spreading Advantages of Guqin Music in the Network Context. It is with a strong professional. Because of the particularity of the music itself, so its website and other sites there is a certain difference, with a specific professional point of view. But in general, the site of the establishment of personnel is professionals in the field, so the uploaded information has a very strong professional. In addition, the construction of the site for the majority of love guqin music creators to provide a platform to show talent, to a certain extent, inspired their creative enthusiasm.

It coverage and spread quickly. Because in the context of the Internet, there will be no carrier of this bondage, so the spread is not just a mesh, and the exponential rate of rise. That is, every recipient of the guqin music will gradually become a new communicator past communicators will continue to spread. In addition, the music network terminals are gradually diversified, covering a number of areas, including the car terminal, corporate terminals, home video terminals and PC, and so on. As a recipient only need a personal computer, you can freely download or listen to guqin music online.

The application is convenient and will not be bound by time and space. Exist in the network resource library which is rich in music information for the guqin professionals to collect material to enhance the level of guqin brought convenience. Moreover, the majority of guqin enthusiasts can also love their own Guqin video and some personal ideas uploaded to the public platform, so that members exchange learning. The convenience of network communication, the arbitrariness of choice and the richness of material have brought many positive effects to the spread of guqin music [3].

The Spreading Drawbacks of Guqin Music in the Network Context. The impact of Guqin music aesthetic. Since ancient times, Guqin music brought the impression that people are self-cultivation, quiet Zhiyuan mood, reflecting the elegant and elegant aesthetic taste. However, the virtual space of the network is under the control of commercial profits to seek development, 
because there is no "threshold", resulting in guqin concert is too popular, and entertainment will be excessive. In the network environment, guqin music will gradually become a natural self-reflection behavior into a commercial performance, piano teaching more attention to the technical level. Especially in the use of some modern forms of popular carrier of the actual process, and even will have many negative effects, guqin culture heritage will not be able to achieve the desired effect.

The inheritance effect gradually weakened. Guqin music performance has evolved from a simple technical performance to the highest aesthetic realm. Past face teacher mentoring is transparent, there is no obstacle to the spread of students can directly experience the master of the music and skills to understand. But in the network environment, although people can access to their own want to get the guqin music therapy, but the accepted is always digital audio and video. This means that the spread of guqin music will be affected by many equipment factors. Such as server effects and propagation speed, and so on. The transparency and immediacy of communication have declined significantly.

The intellectual property rights will be controversial. Although in the network environment, Guqin culture to achieve rapid spread, but the probability of intellectual property disputes have increased significantly. At this stage, to get information resources for everyone who uses the network is a very easy thing. But there are a lot of music copyright received a serious violation, many sites without permission to provide music resources for others to download, and through this piracy infringement from the profit. In the network environment, the spread of guqin gradually expanded, more and more people began to learn and its more and more prominent commodity. China has long been issued a copyright law, cannot adapt to the rapid development of the status of the network to protect the specific needs of the copyright, a serious lack of operational laws and regulations, network transmission infringement events are common.

\section{Conclusion}

To sum up, the efficient transmission of information in this century is inseparable from the network environment, the network in the dissemination and inheritance of music has an important role cannot be ignored. The traditional sense of music transmission will change in the network environment variation. Guqin music is the essence of traditional music in China, so the function of music website must not only be based on the communication function of interest level, but also should play its function of education and information exchange on the basis of protecting the essential attribute of traditional music culture.

\section{Acknowledgements}

Fund Project:"History and Current Situation of Jiangxi Guqin" Jiangxi Province Culture and Arts Scientific Planning Project YG2016179.

\section{References}

[1] Jiang Haoran. Guqin song "Longxiang opera" two versions of the comparison and the spread of guqin music thinking[J]. Music communication, 2016, 10 (1): 34-40.

[2] Yuan Yuan, Hu Heng Luan. Tracing the source of the memory of the ancient Wu - - 2014 years of contemporary Guqin Music Week [J]. People's music, 2015, 12 (5): 55-57.

[3] Liu Chang. On the social and cultural values of Guqin art and its significance in the dissemination of colleges and universities[J]. Popular arts, 2015, 15 (3): 244. 